\title{
Micro-RNA-632 downregulates DNAJB6 in breast cancer
}

\author{
Aparna Mitra, Jack W Rostas, Donna L Dyess, Lalita A Shevde and Rajeev S Samant
}

DNAJB6 is a constitutively expressed member of the HSP40 family. It has been described as a negative regulator of breast tumor progression and a regulator of epithelial phenotype. Expression of DNAJB6 is reported to be compromised with tumor progression. However, factors responsible for its downregulation are still undefined. We used a knowledge-based screen for identifying miRNAs capable of targeting DNAJB6. In this work, we present our findings that hsa-miR-632 (miR632) targets the coding region of DNAJB6. Invasive and metastatic breast cancer cells express high levels of miR-632 compared with mammary epithelial cells. Analysis of RNA from breast tumor specimens reveals inverse expression patterns of DNAJB6 transcript and miR-632. In response to exogenous miR-632 expression, DNAJB6 protein levels are downregulated and the resultant cell population shows significantly increased invasive ability. Silencing endogenous miR-632 abrogates invasive ability of breast cancer cells and promotes epithelial like characteristics noted by E-cadherin expression with concomitant decrease in mesenchymal markers such as Zeb2 and Slug. Thus, miR-632 is a potentially important epigenetic regulator of DNAJB6, which contributes to the downregulation of DNAJB6 and plays a supportive role in malignant progression.

Laboratory Investigation (2012) 92, 1310-1317; doi:10.1038/labinvest.2012.87; published online 18 June 2012

KEYWORDS: DnaJB6; hsa-miR-632

Breast cancer is the leading cancer type for the estimated new cancer cases and second only to lung cancer in the estimated deaths for 2010 in women in the United States. ${ }^{1}$ Years of extensive research has implicated several intrinsic and extrinsic etiologic factors for the initiation and progression of breast cancer. Micro-RNAs are 21-23 nucleotide regulatory RNAs that can target mRNA and cause either transcription or translation repression. ${ }^{2,3}$ In the recent years, micro-RNAs have been emerging as critical epigenetic regulators that modulate almost all stages of breast cancer progression such as proliferation, angiogenesis, invasion, migration, chemoresistance, and stem cell renewal. ${ }^{4-9}$

The focus of our study is DNAJB6 (Mammalian Relative of DNAJ, Mrj), a constitutively expressed member of HSP 40 family. ${ }^{10}$ DNAJB6 gene codes for two spliced variants. The longer spliced variant is referred to as DNAJB6 a $[\operatorname{Mrj}(\mathrm{L})]$ and the shorter is DNAJB6 b $[\operatorname{Mrj}(\mathrm{S})] .{ }^{11}$ Studies on murine DNAJB6 have revealed that it plays a critical role in embryonic development, specifically during chorioallantoic fusion. ${ }^{12}$ This gene product has also been implicated as a player in regulation of cell cycle and mitosis. ${ }^{13}$ Mutations of DNAJB6 are implicated as a potential cause of limb-girdle muscular dystrophy. ${ }^{14,15}$ We have previously reported that
DNAJB6 protein expression is significantly compromised in advanced breast cancers. ${ }^{11}$ Re-expression of DNAJB6a abrogated tumor growth and metastasis in mammary-xenograft studies. ${ }^{11}$ DNAJB6b has been demonstrated to interact with uPAR to increase uPAR-mediated cell adhesion to vitronectin. $^{16}$ Other diseases such as neuro-degenerative disorders involving polyglutamine aggregate may also display a negative outcome if expression or function of DNAJB6 is compromised. ${ }^{17-19}$ However, factors that regulate the expression of this critical protein are yet to be understood. Hence, we undertook an investigation to find out if microRNAs may play a role in downregulating DNAJB6 expression. In this study, we describe that micro-RNA, hsa-miR-632 (referred hereafter as miR-632), downregulates DNAJB6 expression and evaluate its clinical significance.

\section{MATERIALS AND METHODS Cell Culture and Reagents}

MCF10A, MCF10AT, MCF10CA1d.cl.1 (obtained from Barbara Ann Karamanos Cancer Center, Detroit, MI, USA) were grown in DMEM/F12 (Invitrogen, Carslbad, USA) supplemented with $5 \%$ heat-inactivated horse serum, $100 \mathrm{ng} / \mathrm{ml}$ cholera toxin (Calbiochem, San Diego, CA, USA), $10 \mu \mathrm{g} / \mathrm{ml}$ 
insulin (Sigma, St Louis, MO, USA), $25 \mathrm{ng} / \mathrm{ml}$ EGF (Sigma), and $500 \mathrm{ng} / \mathrm{ml}$ hydrocortisone (Sigma). SUM159 cells (purchased from Asterand plc, Detroit, MI, USA) were cultured in Ham's F-12 with $5 \%$ fetal bovine serum supplemented with insulin $(5 \mathrm{mg} / \mathrm{ml})$ and hydrocortisone $(1 \mathrm{mg} / \mathrm{ml})$. MDAMB-231 was grown as described previously. ${ }^{11}$ All cells were maintained in a humidified $5 \% \mathrm{CO}_{2}$ environment at $37^{\circ} \mathrm{C}$.

DNAJB6 rabbit polyclonal (MO1) antibody (Abnova, Taipei City, Taiwan) was used (1:5000) with $5 \%$ milk in PBS containing $0.2 \%$ Tween 20 ). Horseradish peroxidase- $\beta$-actin (Sigma-Aldrich, St Louis, MO, USA) was used at (1:50 000). x-miR:has-mir-632 (cat \# X-miR-8710R) and control mir were obtained from oligoengine ${ }^{\mathrm{TM}}$ (oligoengine, Seattle, WA, USA).

\section{Plasmid Constructs}

Oligos designed to encompass hsa-miR-632 target site in the open reading frame (ORF) of DNAJB6 mRNA 5'agcttttctttgggaatcgaaggggtccccgaggaagcagaagccgagggacggggtc gttttta-3' and $3^{\prime}$-tgatctaaaaacgaccccgtccctcggcttctgcttcctcggg gaccccttcgattcccaaagaa- $5^{\prime}$ were annealed and cloned into HindIII and SpeI sites of pMIR-Report vector (Ambion, Austin, TX, USA) to generate pMIR-Report-DNAJB6. The hsa-miR-632 expression construct was generated in pIRES2EGFP (Clontech, Madison, WI USA) by annealing commercially synthesized oligos corresponding to the mature miRNA hsa-miR-632 5'-ctcgagacggctaccaccacgtcccacaggaag cagacacaaaatggccgacggcctcgttcccegctccgcctcccgtcaagcactgcggt aggaggcg- $3^{\prime}$ and $5^{\prime}$-tgccgatggtggtgcagggtgtccttcgtctgtgttttaccg gctgccggagcaaggggcgaggcggagggcagttcgtgacgccatcctccgcgaattc- $3^{\prime}$ and then cloning them into XhoI and EcoRI sites of pIRES2EGFP.

\section{Transfection}

Cells were transfected with miRNA inhibitor scrambled control clone pEZX-AM01 (CmiR-AN0001-AM01, Gene Copoeia, Rockville, MD, USA) or miRNA inhibitor against hsa-miR-632-pEZX-AM01 (HmiR-AN0742-AM01) using lipofectamine 2000. MCF10AT cells were transfected with pIRES2EGFP-vector or hsa-miR-632-pIRES2EGFP using Fugene 6 (Roche, IN, USA).

\section{Transfection with Anti-miR-632}

To inhibit endogenous miRNA, 50 or $100 \mathrm{nM} \mathrm{x}$-miR-632 or control mir (oligoengine ${ }^{\mathrm{TM}}$ ) was transfected into cells using Lipofectamine 2000. Cells were assayed for knockdown $48 \mathrm{~h}$ post-transfection.

\section{Western Blots}

Cells were transfected with miR-632-pIRES2EGFP or pIRES2EGFP vector alone using Lipofectamine 2000 according to the manufacturer's instructions (Invitrogen). Cells were harvested $42 \mathrm{~h}$ post-transfection in NP-40 lysis buffer. The lysates $(20 \mu \mathrm{g})$ were resolved on SDS-PAGE and transferred onto PVDF membranes. The immunoblot was developed using relevant primary and secondary antibodies as per the respective manufacturer's instructions.

\section{Luciferase Reporter Assay}

Cells were transfected with $50 \mathrm{ng}$ pMIR-Report or pMIRReport-DNAJB6 in combination with either $100 \mathrm{ng}$ pIRES2EGFP vector alone or pIRES2EGFP-miR-632 construct and $25 \mathrm{ng}$ of $\beta$-gal plasmid using with Lipofectamine 2000 (Invitrogen) as stated in the manufacturer's protocol. The assay was terminated $36 \mathrm{~h}$ post-transfection. Luciferase activity was measured using Turner 20/20 luminometer (Turner Biosystems, Sunnyvale, CA). $\beta$-Galactosidase activity was quantitated using Synergy $4^{\mathrm{TM}}$ plate reader (BioTek, Winooski, VT, USA) was used as normalization control.

\section{Quantitative-RT-PCR}

To measure mRNA levels in cell lines or tissues, RNA isolated from cell lines or tissue samples were subjected to qRT-PCR. In all, $1 \mu \mathrm{g}$ of total RNA was used to synthesize cDNA (High Capacity Reverse Transcription Kit, Applied Biosystems, Foster City, CA, USA). PCR was performed using $40 \mathrm{ng}$ of cDNA with respective Taqman primer probe using Bio-Rad $\mathrm{iQ}_{5}$ Real-Time Detection system (Bio-Rad, Hercules, CA, USA). The gene expression $C_{t}$ values of mRNAs from each sample were calculated by normalizing with endorse control, glyceraldehyde-3-phosphate dehydrogenase and relative quantitation values were plotted using GraphPad Prism (La Jolla, CA, USA).

miRNA levels were also analyzed by real time as follows: cDNA was generated using micro-RNA Reverse Transcription kit (Applied Biosystems). Total RNA was used to generate cDNA using primers specific to U6 (control) or has-miR-632. PCR was performed using both U6 (control) or has-miR-632 Taqman primer probes and Taqman Universal Master Mix, No Amperase UNG (Applied Biosystems). The cycling conditions were initial step of $95^{\circ} \mathrm{C}$ for $10 \mathrm{~min}$ followed by 40 cycles of $95^{\circ} \mathrm{C}$ for $15 \mathrm{~s}$ and $60^{\circ} \mathrm{C}$ for $1 \mathrm{~min}$. has-miR-632 miRNA levels were normalized to U6 levels.

\section{Invasion Assay}

Invasion assays were conducted using $8 \mu \mathrm{M}$ polyethylene terpthalate filters (BD Pharmingen), as described earlier. ${ }^{20}$ Cells (transfected with vector or hsa-miR-632) were allowed to invade through matrigel-coated filters for $18 \mathrm{~h}$ in a transwell. Cells invaded to the lower sides of the transwell, were stained using $0.05 \%$ crystal violet, and the cell number was counted as described before. ${ }^{11}$

\section{D Culture}

3D cultures were grown following the protocol by Debnath et $a l^{21}$ Briefly, eight well-chambered cover glass slides (Thermo Scientific, Waltham, MA, USA) were placed on ice and coated with $50 \mathrm{ml}$ of 3D Culture Matrix Basement Membrane Extract Reduced Growth Factor (phenol red free) from Trevigen (Gaithersburg, MD, USA). The slide was 
incubated for $30 \mathrm{~min}$ in $37^{\circ} \mathrm{C}$ incubator. Cells (5000/well, $36 \mathrm{~h}$ post-transfection) were plated in growth media containing $2 \%$ reduced growth factor basement membrane extract (Trevigen). Media was changed every 4 days and the morphology was documented digitally using a Nikon microscope (Nikon, Tokyo, Japan) using the $\times 20$ objective. Laminin V was stained using MAB-D4B5 (Chemicon, USA).

\section{Patient Samples Processing}

Upon obtaining signed consent, surgically excised breast tumor specimens were flash frozen in liquid nitrogen with minimal lag time. Patient tissues were banked per Mitchell Cancer Institute-BioBank protocol (IRB approval \#03-092), before being procured for use (IRB approval \#09-287). All specimens were coded and de-identified. Frozen tissue was grinded using mortar and pestle. Total RNA was isolated using miRNAeasy kit (Qiagen, Valencia, CA, USA) as recommended in the manufacturer's protocol.

\section{RESULTS}

Identification of Potential miRNA Regulators of DNAJB6 miRNAs predicted to target DNAJB6 transcript ( $3^{\prime}$ UTR as well as coding region) were cataloged using the Sanger data base (Table 1). Eleven distinct miRNAs are predicted to target DNAJB6. Out of these five miRNAs are predicted to target the coding region and six are predicted to target the noncoding region ( $\left.3^{\prime} \mathrm{UTR}\right)$. The outcome of this search was manually queried for miRNAs that were reported to be overexpressed in malignancies. Four miRNAs (mir-197, 632, $376 \mathrm{a}$, and $628-3 \mathrm{p}$ ) targeting the coding region and one (mir424) targeting the $3^{\prime}$ UTR were cited in literature to be overexpressed in certain malignancies.

The expression of DNAJB6 has been reported to be highly compromised in invasive (grade III) and metastatic (lymph nodes) breast cancer. ${ }^{11}$ Hence, we queried a set of breast cancer cell lines (viz. MCF10CA1d.cl.1, SUM159, and MDAMB-231) with documented highly invasive and metastatic properties in xenograft studies ${ }^{2-24}$ for the expression of these miRNAs in comparison with MCF10A, an immortalized mammary epithelial cell line (Figure 1a). Tumorigenic but non-invasive and non-metastatic MCF10AT cell line was also used in this comparison. mir-376a and mir-628-3p were not detectable in these cells. Of the three that showed detectable levels, mir-197 expression did not show a distinct trend; if at all there was a slight reduction in expression. However, mir424 and miR-632 expression showed a distinct increase in the three invasive and metastatic cell lines in comparison with MCF10A and MCF10AT. These two miRNAs were evaluated for their capacity to reduce DNAJB6 protein levels by transiently transfecting the respective miRNAs in MCF10A (cell line with high level of DNAJB6). The results showed that miR-632 was able to cause a noticeable reduction in the protein levels of both the spliced variants of DNAJB6 (viz. DNAJB6a and b) (Figure 1b). Digital densitometry analysis shows that there is $74 \%$ reduction in DNAJB6a and 55\% reduction in DNAJB6b (Supplementary Figure 1).

\section{A Putative miR-632 Binding Site Exists in the DNAJB6 ORF}

To validate the miR-632 targeting site from DNAJB6, we cloned the putative binding site of miR-632 (as per Sanger miRNA search software prediction) from the ORF of DNAJB6 in pMIR-REPORT ${ }^{\mathrm{TM}}$ vector to obtain pMIR-REPORT-DNAJB6. This reporter was co-transfected with a construct that constitutively overexpresses mature miR-632 (miR-632-pIRES2EGFP). We found a 90\% reduction in the activity of pMIR-Report-DNAJB6 in MCF10A cells (Figure 2a). These observations provided additional strength to the proposed role of miR-632 in targeting DNAJB6.

miRNAs have several predicted targets and are known to alter expression at transcription or translation or both levels. We tested the effect of miR-632 on DNAJB6 transcript in MCF10A cells. Compared with the non-targeting control, the transcript levels of DNAJB6 in miR-632 transfected cells were reduced by about $40 \%$. Patched 1 (PTCH1) is another predicted target of miR-632. However, the transcript levels of PTCH1 were unaltered in the DNAJB6 expressors (Figure $2 \mathrm{~b})$. This endorsed the specificity of miR-632 in targeting DNAJB6.

To further evaluate the validity of our findings, we used (X-miR-632) to silence the expression of miR-632 from MDA-MB-231 cells. Western blot analysis for DNAJB6 showed that at $50 \mathrm{~nm}$ as well as $100 \mathrm{~nm}$ concentration of XmiR-632 there was a noticeable increase in the levels of both DNAJB6 variants (Figure 2c; Supplementary Figure 2). Cumulatively, these observations confirm the ability of miR-632 to downregulate DNAJB6 expression.

\section{miR-632 Targeting of DNAJB6 Is Conserved Across Species}

Analysis of the predicted target site of miR-632 in the context of the genomic DNA and mRNA sequence of human DNAJB6 gene revealed that it targets a region in exon 5 of the DNAJB6 gene (Figure 3a). As seen in from the complementarity between the seed sequence and the mRNA depicted in Figure 3b, this region is common to both the spliced variants of DNAJB6. Cross-species sequence comparison revealed that mature miR-632 sequence remains fairly unaltered. In fact, it is identical in the primates and deviates minimally in other mammals such as dogs and rodents. Importantly, the mfe (minimum-free energy) of the respective miR-632 hybrid with respective DNAJB6 target sequence remains reasonably comparable (Figure $3 \mathrm{c}$ ). These observations imply that the relationship of the miR-632 in targeting DNAJB6 may be conserved throughout evolution and hence suggestive of the importance of miR-632 as a regulator of DNAJB6. 
Table 1 Micro-RNAs predicted to target DNAJB6 transcript are cataloged using the Sanger database

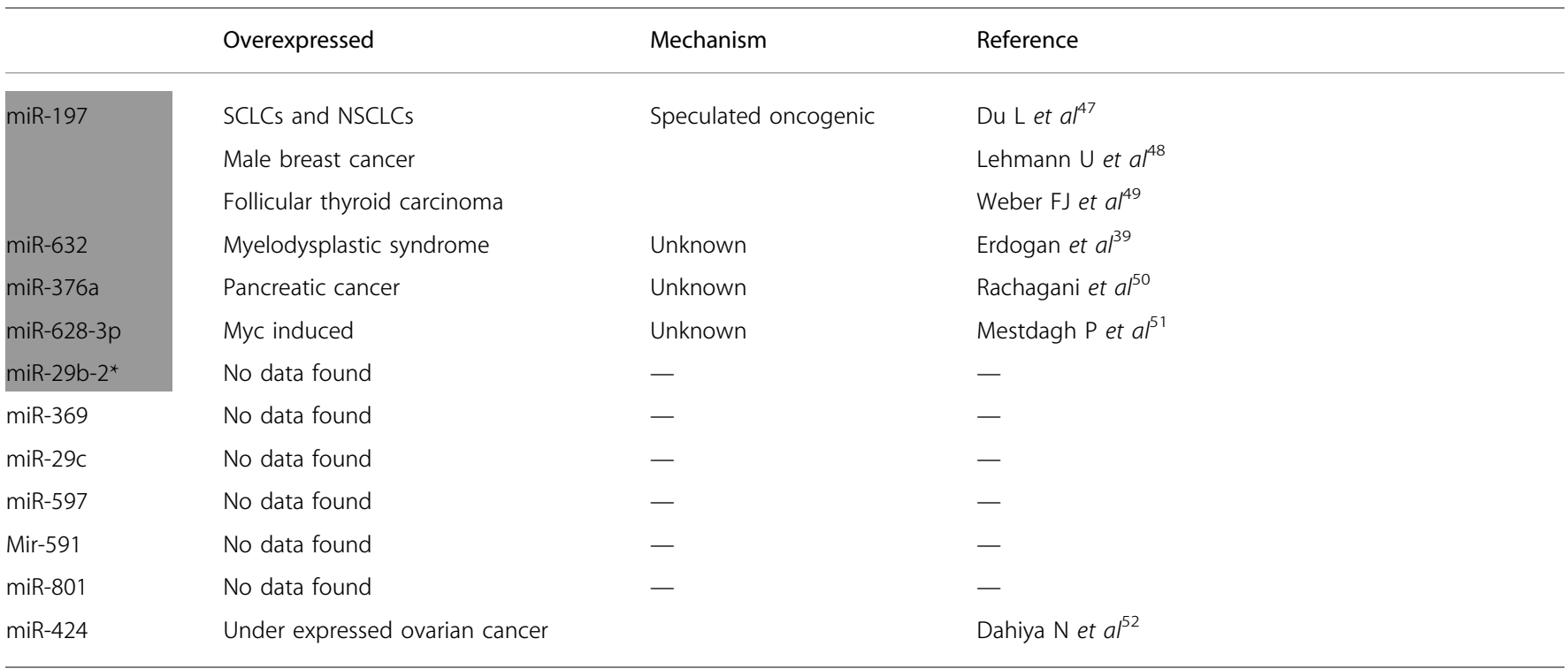

The highlighted miRNAs are predicted to target the coding region of DNAJB6 and the rest are predicted to target the non-coding region ( $\left.3^{\prime} U T R\right)$.

a

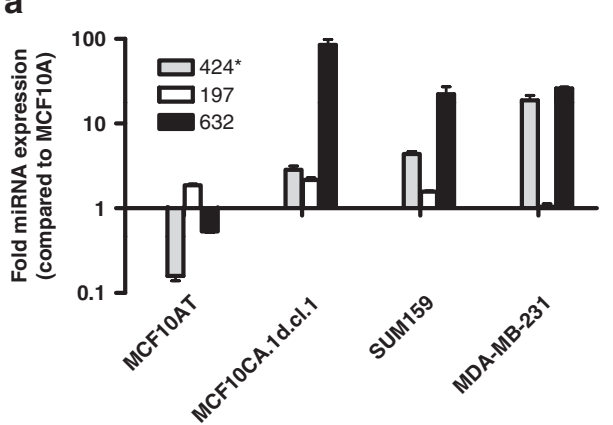

b
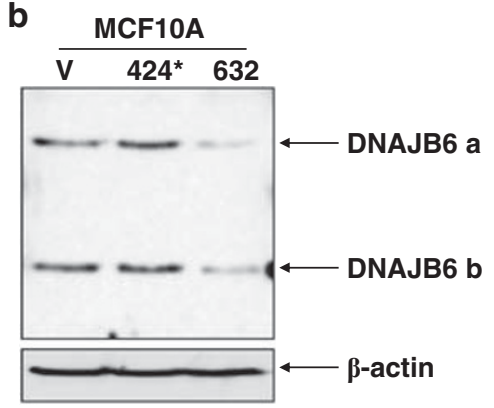

Figure 1 Screening for miRNAs that can potentially target DNAJB6: (a) Levels of each of the miRNAs, miR-197, miR-424, and miR-632 from human breast cancer cell lines MCF10AT, MCF10CA1d.cl.1, SUM159, and MDA-MB-231 were compared with immortalized human mammary epithelial cell line MCF10A using real-time quantitative-PCR. The graph is presented in logarithmic scale for $y$-axis. Reactions were performed in triplicate and the experiment was performed twice. (b) mir-424 and miR-632 were transiently expressed in MCF10A. Total protein extract (20 $\mu \mathrm{g})$ was resolved using SDS-PAGE and subjected to western blot analysis for DNAJB6 levels. $\beta$-Actin level was determined to confirm equal loading.

\section{hsa-miR-632 and DNAJB6 Levels Show Inverse Trends in Breast Cancer Tissues}

We analyzed miR-632 and DNAJB6 levels from RNA harvested from normal breasts tissue and 15 breast tumors. The data show decreased expression of DNAJB6 in cancerous tissues compared with normal breast tissue whereas miR-632 levels were found to be higher in cancerous tissues as compared with the non-cancerous tissues (Figure 4). Thirteen out of 15 tumor specimens showed decreased DNAJB6 transcript and out of those, 12 specimens showed high miR-632 levels relative to normal breast tissue (exact binomial test, $P<0.0001)$.

\section{miR-632 Expression Enhances the Invasive Properties}

We have previously reported that DNAJB6 enhances epithelial attributes and inhibits the mesenchymal phenotype. ${ }^{11,25}$ The mesenchymal phenotype confers invasive ability upon breast cancer cells. ${ }^{26}$ Hence, we analyzed the impact of expression of miR-632 on invasion and levels of mesenchymal markers. MCF10AT breast cancer cells are tumorigenic but non-invasive in nude mice xenograft studies. These cells express very low levels of miR-632 and are known to have high levels of DNAJB6. miR-632 was overexpressed in these cells by transiently transfecting miR-632-pIRES2EGFP. Our results showed that expression of miR-632 caused about a 

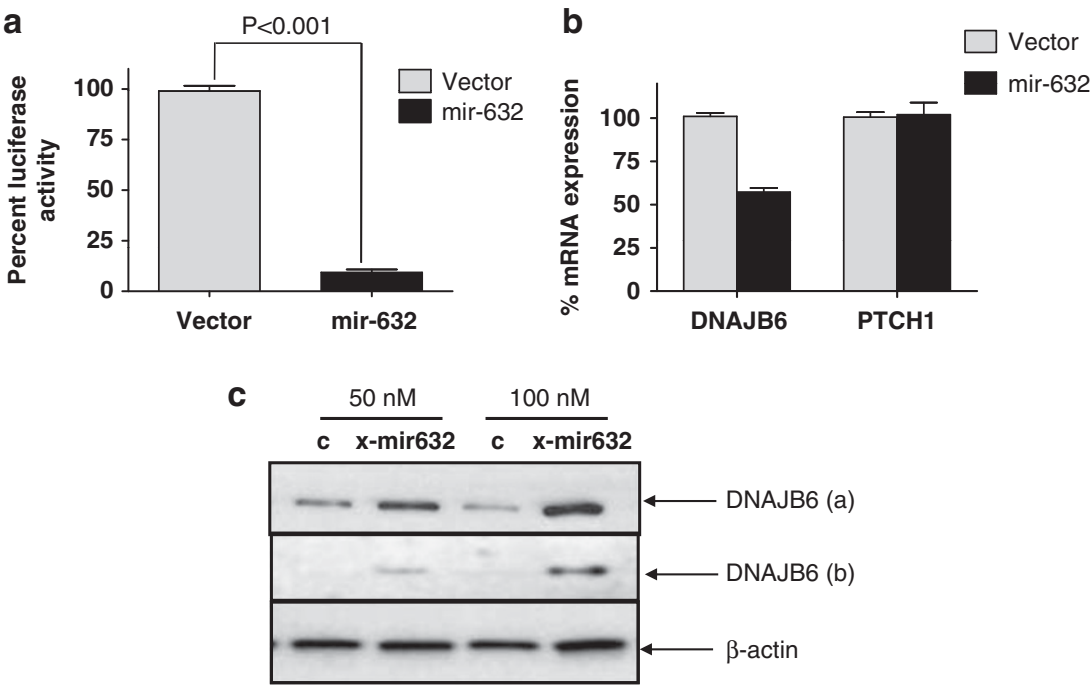

Figure 2 miR-632 targets DNAJB6 for degradation. (a) pMIR-REPORT-DNAJB6 (containing putative binding site of miR-632 from the ORF of DNAJB6) was Cotransfected with miR-632 expression vector, miR-632-pIRES2EGFP, or empty vector control. The assay was performed in triplicate and the experiment was performed twice. The luciferase activity readings were normalized with activity from a co-transfected $\beta$-gal expressing control. The error bars represent standard error of mean (s.e.m.). (b) Levels of DNAJB6 and PTCH1 transcript were evaluated from MCF10A cells treated with miR-632-pIRES2EGFP or empty vector control. GAPDH was used as endorse control. The error bars represent standard error of mean (s.e.m.). The reactions were performed in triplicate and the experiment was performed three times. (c) MDA-MB-231 cells were treated with X-miR-632 or control (50 and $100 \mathrm{nM})$. Total protein extract (30 $\mu \mathrm{g})$ was resolved using SDS-PAGE and subjected to western blot analysis for DNAJB6 levels. $\beta$-Actin levels were determined as loading control.
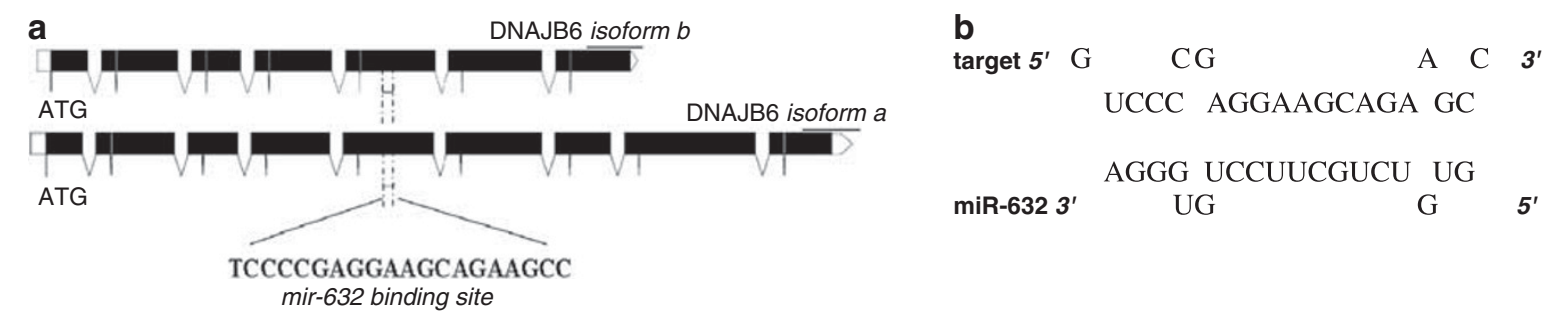

C

\begin{tabular}{|c|c|}
\hline Species & $\begin{array}{c}\text { ID } \\
\text { Mature sequence Accession \# }\end{array}$ \\
\hline Homo sapiens & $\begin{array}{c}\text { hsa-miR-632 } \\
\text { MIMAT0003302 }\end{array}$ \\
\hline Pan troglodytes & $\begin{array}{c}\text { ptr-miR-632 } \\
\text { MIMAT0008289 }\end{array}$ \\
\hline Macaca mulatta & $\begin{array}{c}\text { mml-miR-632 } \\
\text { MIMAT0006477 }\end{array}$ \\
\hline Canis familiaris & $\begin{array}{c}\text { cfa-miR-632 } \\
\text { MIMAT0009923 }\end{array}$ \\
\hline Rattus & $\begin{array}{c}\text { rno-miR-632 } \\
\text { MIMAT0012837 }\end{array}$ \\
\hline
\end{tabular}

\begin{tabular}{|c|c|c|c|c|c|c|c|c|c|c|c|c|c|c|c|c|c|c|c|}
\hline \multicolumn{19}{|c|}{ Mature miRNA sequence } & \multirow{2}{*}{$\begin{array}{c}\begin{array}{c}\mathrm{mfe} \\
\mathrm{kcal} / \mathrm{mol}\end{array} \\
-29.6\end{array}$} \\
\hline $\mathrm{G}$ & U & G & U & C & U & G & C & U & U & C & C & U & G & U & G & G & G & A & \\
\hline G & U & $\mathrm{G}$ & U & C & U & G & C & U & U & C & C & U & G & U & G & G & G & A & -27.1 \\
\hline G & U & G & U & C & U & G & C & U & U & C & C & U & G & U & G & G & G & A & -29.6 \\
\hline G & U & G & U & C & U & G & $\underline{U}$ & U & U & C & C & U & G & U & G & $\mathrm{G}$ & G & A & -26.2 \\
\hline G & U & G & U & C & U & G & $\underline{U}$ & U & U & C & C & U & G & $\underline{c}$ & $\underline{c}$ & G & G & A & -28.3 \\
\hline
\end{tabular}

Figure 3 Analysis of miR-632 binding site from DNAJB6. (a) Diagrammatic representation of miR-632 binding site. Solid black boxes represent exons. (b) Diagrammatic representation of binding of miR-632 seed sequence to its target site in DNAJB6. (c) Cross species sequence comparison miR-632. Highlighted boxes with bold italicized underlined letters depict deviation from human miR-632 sequence. mfe, minimum-free energy.

twofold increase in the invasive ability of the cells (Figure $5 \mathrm{a}(\mathrm{I})$ ). Conversely, we tested the effect of silencing expression of miR-632 in the invasive and metastatic MDA-MB-231 breast cancer cell line. Our observations reveal a $35 \%$ decrease in the invasive ability of these cells upon silencing miR-632 compared with the control-treated cells (Figure $5 b(\mathrm{I})$ ).

Growth in the 3D matrix assays has been accepted to more accurately recapitulates the epithelial organization seen in breast ducts in vivo. ${ }^{27}$ MCF10AT cells transfected with empty 
vector formed well circumscribed, acinar structures. Notably, consistent with the results of the invasion assay, when miR632 was expressed in these cells they formed branched outgrowths indicated with arrows inFigure $5 \mathrm{a}(\mathrm{II})$. Cells embedded in 3D growth environment can produce basement membrane molecules such as laminin $5 .^{28}$ The miR-632 expressing MCF10AT structures retained the ability to deposit

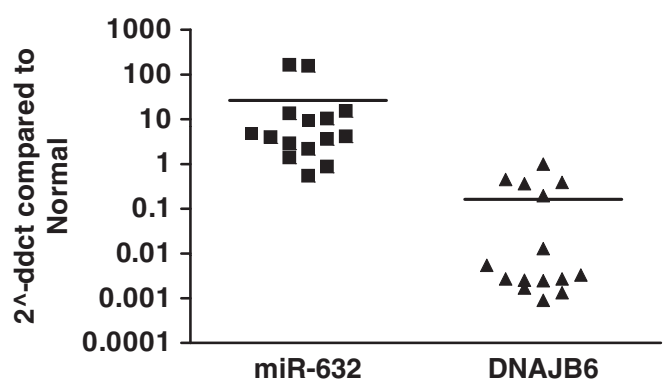

Figure 4 hsa-miR-632 and DNAJB6 levels show inverse trends in breast cancer tissues: Total RNA from 15 breast tumors was analyzed by qRT-PCR for miR-632 and DNAJB6 levels from. The levels were compared with average of normal levels as calibrator, designated as 1 . The graph is presented in logarithmic scale for $y$-axis. basement membrane but exhibited broken (indicated with arrows) laminin 5 around the invasive edge (Supplementary Figure 3).

MDA-MB-231 cells are known to show stellate 3D morphology with projected invasive growths that can link several cell colonies. ${ }^{29}$ Our observations presented inFigure $5 \mathrm{~b}$ (II) indicate that consistent with the results of the invasion assay, upon introduction of anti-mir-632, majority of the cells tend to lose the stellate projections and appear more rounded in 3D culture. Notably, the anti-mir-632 transfected MDA-MB-231 cells showed the presence of intact laminin 5, which was broken (indicated with arrows) in the control anti-mir transfected cells (Supplementary Figure 3).

Cumulatively, these observations indicate a gain in invasive-mesenchymal like cellular morphology in the presence of miR-632. Expression analysis using qRT-PCR revealed that X-miR-632 treatment downregulated mesenchymal markers Zeb2 and SLUG, whereas epithelial marker, E-cadherin was upregulated. These observations are consistent with our previous report of epithelial-mesenchymal transition (EMT) reversal seen in DNAJB6 expressors are consistent with our previous report of EMT reversal seen in DNAJB6 expressors (Supplementary Figure 4). a i

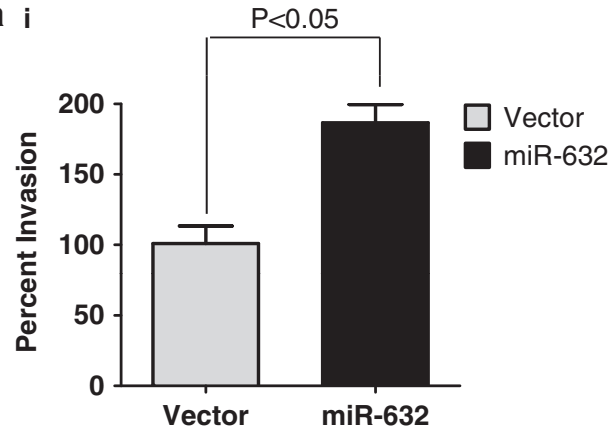

b i

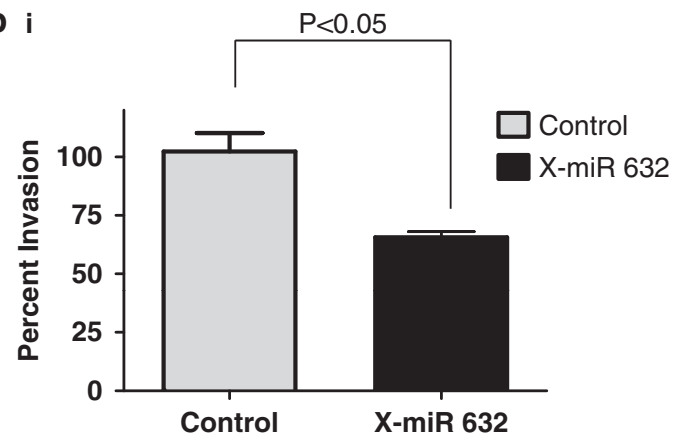

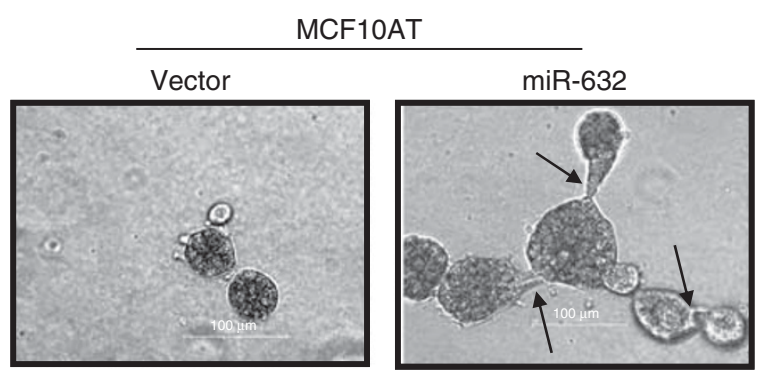

ii

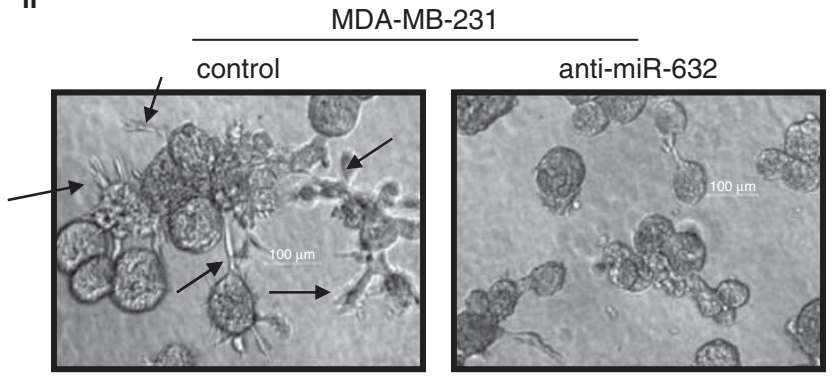

Figure 5 miR-632 expression enhances the invasive properties. (ai) MCF10AT cells were transiently transfected miR-632-pIRES2EGFP and were allowed to invade through matrigel-coated filters for $18 \mathrm{hrs}$. Invaded cells were visualized using crystal violet, and the cell number was counted and compared with invasion of cells transfected with empty vector control. (aii) MCF10AT cells were transiently transfected with empty pIRES2EGFP vector or miR-632pIRES2EGFP and their growth in 3D was assessed. Arrows point the invasive outgrowth. Experiments were performed in triplicate and the experiment was performed two times. (bi) MDA-MB-231 cells were treated with X-miR-632 $(100 \mathrm{nM})$ or the control and the invasion assay was performed as described before. (bii) MDA-MB-231 cells were transfected with miRNA inhibitor scrambled control clone pEZX-AM01 (control) or anti-miR-632-pIRES2EGFP and their growth in $3 \mathrm{D}$ was assessed. Arrows point the invasive outgrowth. Experiments were performed in triplicate and the experiment was performed two times. 


\section{DISCUSSION}

Differential regulation of miRNA in cancers of diverse types is known. Many human miRNA genes are located at genomic loci associated with cancer, suggesting the role of miRNAs in cancer. ${ }^{30}$ The role of miRNAs in breast cancer cell growth and metastasis has been widely accepted. ${ }^{7,31-35}$ miRNAs have emerged as novel candidates markers and treatment targets in breast cancer and have a huge potential of evolving into effective biomarkers because of their stability and availability for detection. ${ }^{36-38}$

However, newer miRNAs are still being discovered and validated for their activity. To date, the role of miR-632 in cancer has not been described. Also, no specific target has been validated for this miRNA. Notably, the importance of miR-632 is getting realized. Recent interesting studies by Erdogan et al $^{39}$ revealed that expression level of miR-632 is positively associated with myelodysplastic syndromes (MDS) and has high discrimination ability compared with normal. Cancer for the most part is associated with aging; hence it is interesting to note that an aging-associated disorder such as MDS shows upregulation of miR-632. One other detailed study involving analysis of exosomes secreted by immune cells revealed high miR-632 levels in the exosomes. ${ }^{40}$ This study shows that miRNAs transferred during immune synapsis are able to modulate gene expression in recipient cells. Thus, it is conceivable that DNAJB6 expression in the breast epithelial cells could also be modulated by miR-632 delivered through exosomes in the microenvironment.

The phenomenon of EMT has been implied as a determinant of the invasiveness of solid tumors. ${ }^{4-43}$ Mesenchymal characteristics are responsible for the invasive ability of breast cancer cells. ${ }^{44-46}$ Findings from our laboratory have indicated that loss of DNAJB6 expression is concurrent with tumor progression and may contribute to mesenchymal like properties. ${ }^{11,25}$ Our work presented here identifies miR-632 as a potential negative regulator of DNAJB6 expression. Overexpression of miR-632 in MCF10AT lead to increased invasion, conversely, silencing miR-632 reduced invasive ability and mesenchymal markers. These observations are consistent with its action of reducing DNAJB6 expression. Our observations show that miR-632 is capable of silencing both spliced variants of DNAJB6. However, the extent of silencing is variable and this may reflect as differential interactions of miR-632 with the spliced variants. Interestingly, immunohistochemical analysis of DNAJB6 in IDC grade III and in lymph node metastasis of breast cancer shows a total loss of DNAJB6 protein signal, which is expected if both the spliced variants are abrogated. There could be multiple negative regulators of DNAJB6 expression that may be responsible for abrogating DNAJB6 expression and based on observation presented here, we propose that miR-632 is one of the negative regulators of DNAJB6 expression.

Supplementary Information accompanies the paper on the Laboratory Investigation website (http://www.laboratoryinvestigation.org)

\section{ACKNOWLEDGEMENTS}

USPHS Grants CA140472 (RS Samant) and CA138850 (LA Shevde). J Rostas is a recipient of the American Medical Association Seed Grant 2011. RS

Samant is the recipient of the Mayer Mitchell Award for Excellence in Cancer Research and acknowledges the support.

\section{DISCLOSURE/CONFLICT OF INTEREST}

The authors declare no conflict of interest.

1. Jemal A, Siegel $R, X u J$, et al. Cancer statistics. CA Cancer J Clin 2010;60:277-300.

2. Bartel DP. MicroRNAs: genomics, biogenesis, mechanism, and function. Cell 2004;116:281-297.

3. Gebauer F, Hentze MW. Molecular mechanisms of translational control. Nat Rev Mol Cell Biol 2004;5:827-835.

4. Dumont N, Tlsty TD. Reflections on miR-ing effects in metastasis. Cancer Cell 2009;16:3-4.

5. Rameshwar P. Potential novel targets in breast cancer. Curr Pharm Biotechnol 2009;10:148-153.

6. Wright JA, Richer JK, Goodall GJ. microRNAs and EMT in mammary cells and breast cancer. J Mammary Gland Biol Neoplasia 2010;15:213-223.

7. O'Day E, Lal A. MicroRNAs and their target gene networks in breast cancer. Breast Cancer Res 2010;12:201.

8. Yu Z, Baserga R, Chen $L$, et al. microRNA, cell cycle, and human breast cancer. Am J Pathol 2010;176:1058-1064.

9. Calin GA, Croce CM. MicroRNA-cancer connection: the beginning of a new tale. Cancer Res 2006;66:7390-7394.

10. Mitra A, Shevde LA, Samant RS. Multi-faceted role of HSP40 in cancer. Clin Exp Metastasis 2009;26:559-567.

11. Mitra A, Fillmore RA, Metge BJ, et al. Large isoform of MRJ (DNAJB6) reduces malignant activity of breast cancer. Breast Cancer Res 2008;10:R22.

12. Hunter PJ, Swanson BJ, Haendel MA, et al. Mrj encodes a DnaJ-related co-chaperone that is essential for murine placental development. Development 1999;126:1247-1258.

13. Dey S, Banerjee P, Saha P. Cell cycle specific expression and nucleolar localization of human J-domain containing co-chaperone Mrj. Mol Cell Biochem 2009;322:137-142.

14. Sarparanta J, Jonson PH, Golzio C, et al. Mutations affecting the cytoplasmic functions of the co-chaperone DNAJB6 cause limb-girdle muscular dystrophy. Nat Genet 2012;44:450-455.

15. Harms $M B$, Sommerville RB, Allred $P$, et al. Exome sequencing reveals DNAJB6 mutations in dominantly-inherited myopathy. Ann Neurol 2012;71:407-416.

16. De Bock $C E$, Lin $\mathrm{Z}$, Mekkawy $\mathrm{AH}$, et al. Interaction between urokinase receptor and heat shock protein MRJ enhances cell adhesion. Int J Oncol 2010;36:1155-1163.

17. Fayazi Z, Ghosh S, Marion S, et al. A Drosophila ortholog of the human MRJ modulates polyglutamine toxicity and aggregation. Neurobiol Dis 2006;24:226-244.

18. Chuang JZ, Zhou H, Zhu M, et al. Characterization of a brain-enriched chaperone, MRJ, that inhibits Huntingtin aggregation and toxicity independently. J Biol Chem 2002;277:19831-19838.

19. Durrenberger PF, Filiou MD, Moran LB, et al. DnaJB6 is present in the core of Lewy bodies and is highly up-regulated in parkinsonian astrocytes. J Neurosci Res 2009;87:238-245.

20. Menezes ME, Mitra A, Shevde LA, et al. DNAJB6 governs a novel regulatory loop determining Wnt/beta-catenin signaling activity. Biochem J 2012;444:573-580.

21. Debnath J, Muthuswamy SK, Brugge JS. Morphogenesis and oncogenesis of MCF-10A mammary epithelial acini grown in threedimensional basement membrane cultures. Methods 2003;30:256-268.

22. Morrow KA, Das $\mathrm{S}$, Metge BJ, et al. Loss of tumor suppressor merlin in advanced breast cancer is due to post-translational regulation. J Biol Chem 2011;286:40376-40385.

23. Harris LG, Pannell LK, Singh S, et al. Increased vascularity and spontaneous metastasis of breast cancer by hedgehog signaling mediated upregulation of cyr61. Oncogene; advance online publication, 7 November 2011; doi:10.1038/onc.2011.496; PMID: 22056874.

24. Das S, Harris LG, Metge BJ, et al. The hedgehog pathway transcription factor GLI1 promotes malignant behavior of cancer cells by upregulating osteopontin. J Biol Chem 2009;284:22888-22897. 
25. Mitra A, Menezes ME, Shevde LA, et al. DNAJB6 induces degradation of beta-catenin and causes partial reversal of mesenchymal phenotype. J Biol Chem 2010;285:24686-24694.

26. Guarino M, Rubino B, Ballabio G. The role of epithelial-mesenchymal transition in cancer pathology. Pathology 2007;39:305-318.

27. Jacks T, Weinberg RA. Taking the study of cancer cell survival to a new dimension. Cell 2002;111:923-925.

28. Zahir N, Lakins JN, Russell A, et al. Autocrine laminin-5 ligates alpha6beta4 integrin and activates RAC and NFkappaB to mediate anchorage-independent survival of mammary tumors. J Cell Biol 2003;163:1397-1407.

29. Kenny PA, Lee GY, Myers CA, et al. The morphologies of breast cancer cell lines in three-dimensional assays correlate with their profiles of gene expression. Mol Oncol 2007;1:84-96.

30. Calin GA, Sevignani C, Dumitru CD, et al. Human microRNA genes are frequently located at fragile sites and genomic regions involved in cancers. Proc Natl Acad Sci USA 2004;101:2999-3004.

31. Iorio MV, Ferracin M, Liu CG, et al. MicroRNA gene expression deregulation in human breast cancer. Cancer Res 2005;65:7065-7070.

32. Ma L, Teruya-Feldstein J, Weinberg RA. Tumour invasion and metastasis initiated by microRNA-10b in breast cancer. Nature 2007;449:682-688.

33. Hurst DR, Edmonds MD, Welch DR. Metastamir: the field of metastasisregulatory microRNA is spreading. Cancer Res 2009;69:7495-7498.

34. Pfeffer $U$, Romeo F, Noonan DM, et al. Prediction of breast cancer metastasis by genomic profiling: where do we stand? Clin Exp Metastasis 2009;26:547-558.

35. Shi M, Liu D, Duan $\mathrm{H}$, et al. Metastasis-related miRNAs, active players in breast cancer invasion, and metastasis. Cancer Metastasis Rev 2010;29:785-799.

36. Andorfer CA, Necela BM, Thompson EA, et al. MicroRNA signatures: clinical biomarkers for the diagnosis and treatment of breast cancer. Trends Mol Med 2011;17:313-319.

37. Greene SB, Herschkowitz JI, Rosen JM. Small players with big roles: microRNAs as targets to inhibit breast cancer progression. Curr Drug Targets 2010;11:1059-1073.

38. Heneghan HM, Miller N, Kerin MJ. MiRNAs as biomarkers and therapeutic targets in cancer. Curr Opin Pharmacol 2010;10:543-550.

39. Erdogan B, Facey C, Qualtieri J, et al. Diagnostic microRNAs in myelodysplastic syndrome. Exp Hematol 2011;39:915-926 e912.
40. Mittelbrunn $M$, Gutierrez-Vazquez $C$, Villarroya-Beltri $C$, et al. Unidirectional transfer of microRNA-loaded exosomes from T cells to antigen-presenting cells. Nat Commun 2011;2:282.

41. Nguyen DX, Bos PD, Massague J. Metastasis: from dissemination to organ-specific colonization. Nat Rev Cancer 2009;9:274-284.

42. Hanahan D, Weinberg RA. Hallmarks of cancer: the next generation. Cell 2011;144:646-674.

43. May CD, Sphyris N, Evans KW, et al. Epithelial-mesenchymal transition and cancer stem cells: a dangerously dynamic duo in breast cancer progression. Breast Cancer Res 2011;13:202.

44. Creighton CJ, Chang JC, Rosen JM. Epithelial-mesenchymal transition (EMT) in tumor-initiating cells and its clinical implications in breast cancer. J Mammary Gland Biol Neoplasia 2010;15: 253-260.

45. Blick $T$, Widodo $E$, Hugo $H$, et al. Epithelial mesenchymal transition traits in human breast cancer cell lines. Clin Exp Metastasis 2008;25:629-642.

46. Micalizzi DS, Farabaugh SM, Ford HL. Epithelial-mesenchymal transition in cancer: parallels between normal development and tumor progression. J Mammary Gland Biol Neoplasia 2010 15:117-134.

47. Du L, Schageman JJ, Subauste MC, et al. miR-93, miR-98, and miR-197 regulate expression of tumor suppressor gene FUS1. Molecular Cancer Res 2009;7:1234-1243.

48. Lehmann U, Streichert T, Otto B, Albat C, Hasemeier B, Christgen $\mathrm{H}_{\text {, }}$ et al. Identification of differentially expressed microRNAs in human male breast cancer. BMC Cancer 2010;10:109.

49. Weber F, Teresi RE, Broelsch CE, et al. A limited set of human MicroRNA is deregulated in follicular thyroid carcinoma. J Clin Endocrinol Metab 2006;91:3584-3591.

50. Rachagani S, Kumar S, Batra SK. MicroRNA in pancreatic cancer: pathological, diagnostic and therapeutic implications. Cancer Letters 2010;292:8-16.

51. Mestdagh P, Fredlund E, Pattyn F, et al. MYCN/c-MYC-induced microRNAs repress coding gene networks associated with poor outcome in MYCN/c-MYC-activated tumors. Oncogene 2010;29: 1394-1404.

52. Dahiya N, Sherman-Baust CA, Wang TL, et al. MicroRNA expression and identification of putative miRNA targets in ovarian cancer. PloS One 2008;3:e2436. 